\title{
Characterization of the monolithic fiber amplifier engineering prototype for the next generation of gravitational wave detectors
}

Wellmann, Felix, Steinke, Michael, Thies, Fabian, Bode, Nina, Oppermann, Patrick, et al.

Felix Wellmann, Michael Steinke, Fabian Thies, Nina Bode, Patrick Oppermann, Benno Willke, Ludger Overmeyer, Jörg Neumann, Dietmar Kracht, "Characterization of the monolithic fiber amplifier engineering prototype for the next generation of gravitational wave detectors," Proc. SPIE 10897, Fiber Lasers XVI: Technology and Systems, 1089722 (7 March 2019); doi: $10.1117 / 12.2508532$ 


\title{
Characterization of the Monolithic Fiber Amplifier Engineering Prototype for the Next Generation of Gravitational Wave Detectors
}

\author{
Felix Wellmann ${ }^{1,}$, Michael Steinke ${ }^{1}$, Fabian Thies ${ }^{2}$, Nina Bode $^{2}$, Patrick Oppermann $^{2}$, \\ Benno Willke ${ }^{2}$, Ludger Overmeyer ${ }^{1,3}$,Jörg Neumann ${ }^{1}$, Dietmar Kracht ${ }^{1}$ \\ 1. Laser Zentrum Hannover e.V., Hollerithallee 8, 30419 Hannover, Germany \\ 2. Max-Planck-Institut für Gravitationsphysik (Albert-Einstein-Institut) and Leibniz Universität Hannover, \\ Callinstr. 38, 30167 Hannover, Germany \\ 3. Institut für Transport- und Automatisierungstechnik, Leibniz Universität Hannover, An der Universität 2, 30823 Garbsen, Germany \\ *f.wellmann@lzh.de
}

\begin{abstract}
Single-frequency $\mathrm{Yb}^{3+}$ fiber amplifiers operating at $1064 \mathrm{~nm}$ are promising candidates to fulfill the challenging requirements for laser sources of the next generation of interferometric gravitational wave detectors. We present the current development progress of a fiber amplifier engineering prototype and compare the optical and thermal performance to the solid-state-laser source of advanced LIGO. The fiber amplifier system consists of two monolithic fiber amplifier stages which currently deliver more than $110 \mathrm{~W}$ (functional prototype demonstrated $215 \mathrm{~W}$ [9,11]) of output power. The fiber amplifier output beam has one to two orders of magnitude lower relative beam pointing and relative power noise in the lower frequency range of $1 \mathrm{~Hz}$ to $100 \mathrm{~Hz}$ compared to the solid-state-laser system. It also has a polarization extinction ratio above $21 \mathrm{~dB}$ and a $\mathrm{TEM}_{00}$-mode content of more than $97.8 \% \pm 0.6 \%$ at $110 \mathrm{~W}$ output power. Besides the optical properties, repair and maintenance procedures are improved by a modular design of the system. Each of the modules can separately be maintained and repaired or easily be replaced by a preassembled module; it therefore minimizes laser downtimes. Another advantage is the lower heat load of approximately $500 \mathrm{~W}$ compared to the SSL, which produces more than $4500 \mathrm{~W}$ of heat, both at an optical output power of $200 \mathrm{~W}$. The lower heat load simplifies cooling and reduces the complexity of the modules.
\end{abstract}

Keywords: monolithic fiber amplifier, single-frequency, gravitational wave detection

\section{INTRODUCTION}

At the beginning of 2016, the first direct observation of gravitational waves by an interferometric gravitational wave detector (GWD) has been reported [1]. Several further detections, partly confirmed by independent observations in the electromagnetic spectrum [2], led to an increased interest in a new generation of GWDs with increased sensitivity. Several design studies have been compiled [3,4] and propose GWDs that are particularly more sensitive at lower frequencies, which would open a new range of detectable astrophysical events. Two different design approaches that directly impact the laser development are discussed: Operating the interferometer optics at room temperature with increased laser power at $1064 \mathrm{~nm}$ or operation at cryogenic temperatures with laser systems at around $1550 \mathrm{~nm}$. The GWD design using room temperature interferometer optics increases sensitivity by operating at higher laser power of up to $1 \mathrm{~kW}$.

Current state-of-the-art GWDs, i.e., the advanced LIGO (aLIGO) detectors, use solid-state injection-locked Nd:YAG ring oscillators as high-power laser [5]. These solid-state-lasers (SSL) fulfill the power and noise requirements but have certain disadvantages regarding usability, maintenance procedures and further power scaling. At increased output power levels, these lasers require complex cooling strategies and tend to increased thermal lensing, which leads to instabilities and reduction of beam quality. System inherently, the output power cannot be freely chosen because the thermal lensing is part of the resonator design and is highly optimized for a pre-defined working point. The complexity of the SSL system and its cooling system makes maintenance and repair difficult.

Fiber technology has been identified as a promising alternative to overcome limitations of the current laser system. Fiber amplifiers enable very high output power levels and fulfill GWD requirements regarding low noise levels and exceptional beam quality (above $90 \% \mathrm{TEM}_{00}$ content). In addition to their optical performance, fiber amplifiers are less affected by environmental condition fluctuations and produce lower and better distributed heat load, which make the

Fiber Lasers XVI: Technology and Systems, edited by Adrian L. Carter, Proc. of SPIE Vol. 10897, $1089722 \cdot$ (c) 2019 SPIE · CCC code: $0277-786 X / 19 / \$ 18 \cdot$ doi: $10.1117 / 12.2508532$ 
systems less complex. The reduced system complexity simplifies maintenance procedures, component upgrades, and repair. Therefore, high power monolithic fiber amplifier at $1064 \mathrm{~nm}$ that emit the linearly-polarized fundamental TEM 00 mode are promising candidates and are thus further investigated.

In recent years, several monolithic single-frequency fiber amplifiers have been developed that demonstrated the power scalability of such devices. In 2008 Mermelstein et al. showed $194 \mathrm{~W}$ output power [6], in $2013 \mathrm{Ma}$ et al. up to $332 \mathrm{~W}$ output power [7] and recently Huang et al. demonstrated an output power of $414 \mathrm{~W}$ [8]. These amplifiers used $\mathrm{Yb}^{3+}$-doped large-mode-area (LMA) fibers to increase the threshold of stimulated Brillouin scattering (SBS), which is one of the predominant power scaling limitations of single-frequency fiber amplifiers.

We developed a fiber amplifier prototype particularly optimized for the use in GWDs and characterized it according to their requirements. We demonstrated, besides the power scaling capabilities, the preservation of low noise properties and excellent beam quality. Our quasi-monolithic fiber amplifier system operating at $1064 \mathrm{~nm}$ delivers an output power of up to $215 \mathrm{~W}$ [9,11], without the onset of SBS. The setup consists of a narrow linewidth seed source with $2 \mathrm{~W}$ output power that is afterward amplified in two fiber amplifier stages to $15 \mathrm{~W}$ and then to $215 \mathrm{~W}$. Both amplification stages use double-clad (DC) $\mathrm{Yb}^{3+}$-doped LMA fibers. Recently, we focused our work on an engineering fiber amplifier prototype with increased reliability and usability.

\section{SINGLE-FREQUENCY HIGH POWER FIBER AMPLIFIER AT 1064 NM}

Our fiber amplifier engineering prototype (c.f. Figure 1) consists of three individual modules, the seed-laser module, the first (pre-amplifier) amplifier module, and the second (main-amplifier) amplifier module. The modular design improves the usability, maintenance, and repair procedures. Each module is spatially separated from the other to give the system a more explicit arrangement, which improves error diagnostics procedures. Besides, in case of a module failure, a redundant module can replace the defective module. The modules are either connected using $1 / 2$ " fiber end-caps (between pre- and main-amplifier module) or a SMA-connector with an integrated cladding light stripper between the seed-laser module and the pre-amplifier. A plug-in design enables easy replacement of a module by a preassembled module or an upgraded version. This replacement is done without redoing splices between PM-LMA fibers. Therefore, expensive downtimes because of laser failure or maintenance can be kept at a minimum.

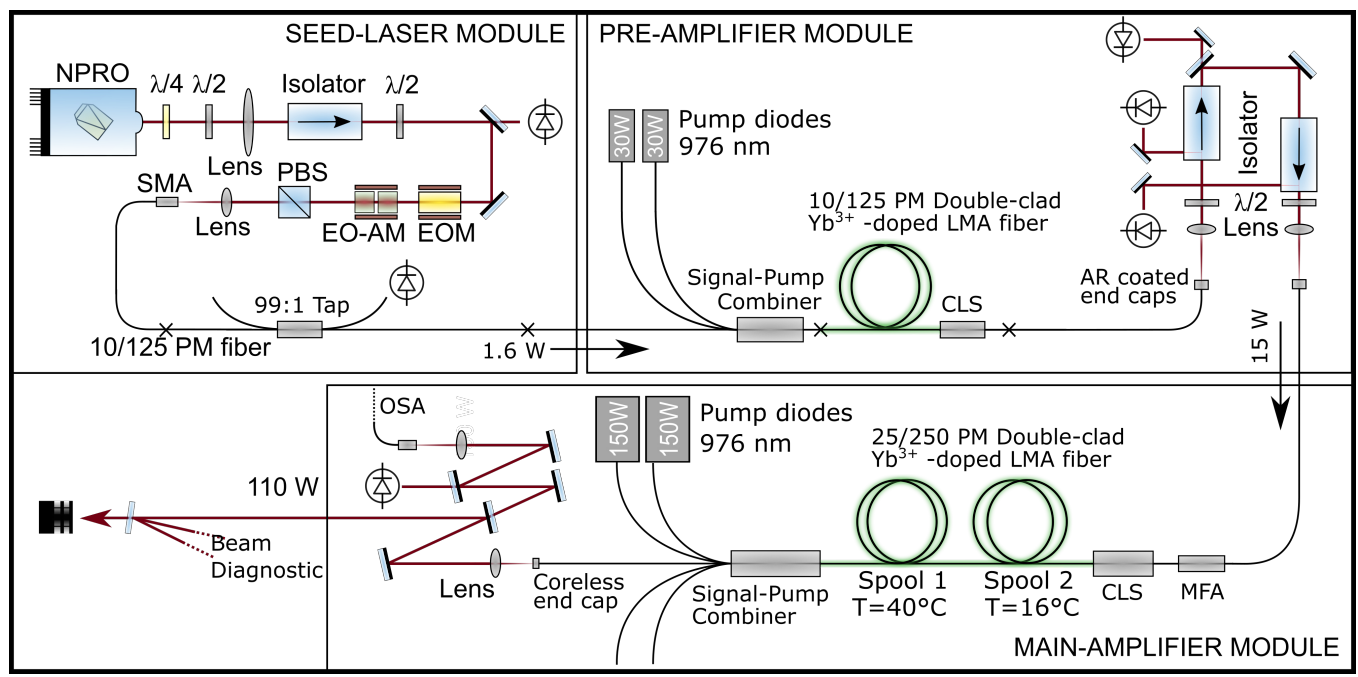

Figure 1: Schematic of the fiber amplifier: The system consists of three modules: First module: NPRO as a seed source, free space optics for mode-matching into a PM-10/125 fiber, an isolator for seed protection and phase/intensity modulators for stabilization purposes. This stage delivers $1.6 \mathrm{~W}$ at $1064 \mathrm{~nm}$. Second module: The pre-amplifier stage consists of a monolithic fiber amplifier based on an $\mathrm{Yb}^{3+}$-doped PM 10/125 fiber, which is pumped at $976 \mathrm{~nm}$. This stage delivers $15 \mathrm{~W}$ of output power and is protected by two high power isolators. It has also multiple ports for monitoring the pre-amplifier output power, backward propagating light from the main-amplifier and PER measurements. Third module: The main-amplifier is based on a $\mathrm{Yb}^{3+}$-doped PM $25 / 250$ fiber that is also

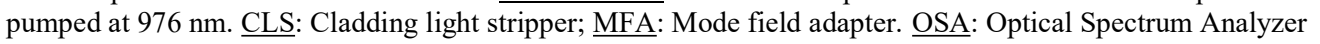


The first stage comprises a non-planar ring oscillator (NPRO) as seed laser, an optical isolator to protect the seed source from back-reflections, and optics to mode-match the laser beam into a PM 10/125 fiber. The first stage provides the equipment to monitor power fluctuations and variations of the polarization. In case of a seed source failure, the NPRO can easily be replaced, and its alignment to the other optical components is mechanically kept within sufficient tolerances to improve realignment. The module provides $1.6 \mathrm{~W}$ out of the fiber, which is sufficient to saturate the first amplifier stage. The particular NPRO seed source technology has proven over the last decade to show exceptional power and frequency noise properties and reliability. However, its output power is limited to a maximum of $2 \mathrm{~W}$, which is not sufficient for the use in GWDs; therefore, the power is amplified in two subsequent amplification modules.

The pre-amplifier stage is based on a LMA $\mathrm{Yb}^{3+}$-doped 10/125 PM fiber that is pumped at $976 \mathrm{~nm}$ in a copropagating configuration. This configuration reduces load on critical components such as the signal-pump-combiner but

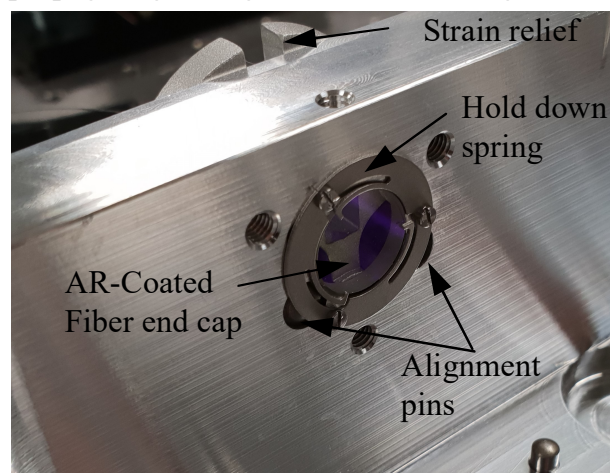

Figure 2: 1/2" AR coated fiber end cap mounted in a kinematic mount. has the disadvantage of a decreased SBS threshold (compared to a counter-pumped configuration). Any residual pump light is removed by an in-house fabricated cladding light stripper (CLS) that uses a microstructured cladding [10]. The seed signal is amplified by the pre-amplifier to a maximum power of $15 \mathrm{~W}$. The amplifier is protected from backward propagating light coming from the mainamplifier by two optical isolators. The module is connected to the main-amplifier by $1 / 2 "$ AR-coated fiber endcaps as shown in Figure 2. The fibers are directly fused to the substrate using a $\mathrm{CO}_{2}$-Laser. These endcaps are designed for high power operation and are kinematically mounted to ensure stable operation and easy replacement of components. In addition, they do not degrade the beam quality.

The second amplifier stage is seeded by the pre-amplifier and is counter-propagating pumped at $976 \mathrm{~nm}$. This configuration increases the SBS threshold by a more advantageous temperature distribution along the fiber [13]. The amplifier has currently an output power of $110 \mathrm{~W}$ out of a LMA Yb ${ }^{3+}$-doped 25/250 PM fiber. Figure 3 a) shows the slope up to $110 \mathrm{~W}$ with a slope efficiency of $67 \% \pm 2 \%$ at a pump power of $148 \mathrm{~W}$. The $110 \mathrm{~W}$ output power is achieved by operating only one of the two pump diodes (c.f. Figure 1). The second pump diode will enable output power levels of up to $200 \mathrm{~W}$. The low efficiency at amplifier output power level below $40 \mathrm{~W}$ is caused by a central wavelength of the pump shifted away from the absorption maximum of $\mathrm{Yb}^{3+}$ at $976 \mathrm{~nm}$. A stable polarization extinction ratio (PER) of above $21 \mathrm{~dB}$ has been measured over the entire amplifier output range (Figure $3 \mathrm{~b}$ )).
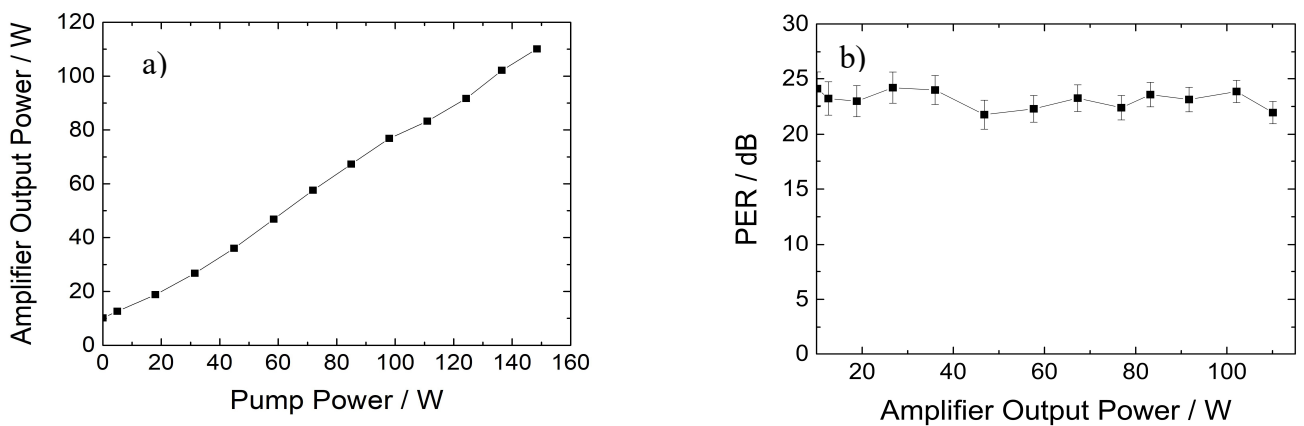

Figure 3: a) Slope of the main-amplifier module with a maximum output power of $110 \mathrm{~W}$ at $148 \mathrm{~W}$ pump power; Slope efficiency is approximately $67 \% \pm 5 \%$. One pump diode was operated to achieve $110 \mathrm{~W}$ amplifier output power. b) The PER over the entire output power range is above $21 \mathrm{~dB}$.

In addition to the PER measurements the beam quality has been characterized by analyzing the modal content of the beam. A linear polarization and a high $\mathrm{TEM}_{00}$-mode content are necessary for the use of the amplifier in GWDs because the wrong polarization and higher order modes are removed by polarizing optics and a mode-cleaner before the beam is coupled into the interferometer. The beam quality, i.e., the fundamental $\mathrm{TEM}_{00}$ mode content was analyzed with a nonconfocal scanning ring cavity as described in [14]. The eigenmodes of such a ring cavity correspond to the TEM modes 
of the same order. Thus, scanning the cavity over a free spectral range (FSR) allows measuring the relative TEM00 mode content and the higher order mode content. Figure 4 a) shows a scan over a FSR of the cavity while the main amplifier is operated at $110 \mathrm{~W}$ output power. The signal at FSR equal 0 and 1 corresponds to the TEM00 mode and the peaks in between are caused by high order modes. The fundamental mode content is calculated by comparing the fundamental mode fit (Figure 4 a) Fundamental Fit) to the overall fit of the scan (Figure 4 a) Overall Fit). The scan at $110 \mathrm{~W}$ corresponds to a TEM00 mode content of $97.8 \% \pm 0.6 \%$. The fundamental mode content is above $97 \%$ for the entire main-amplifier slope (Figure $4 \mathrm{~b}$ ).

The excellent optical properties are achieved by the integration of the pump combiner [12], the CLS and the mode field adapter (MFA) directly in the active fiber. Therefore, additional splices have been avoided, which are usually the reason for a reduced PER and beam quality.
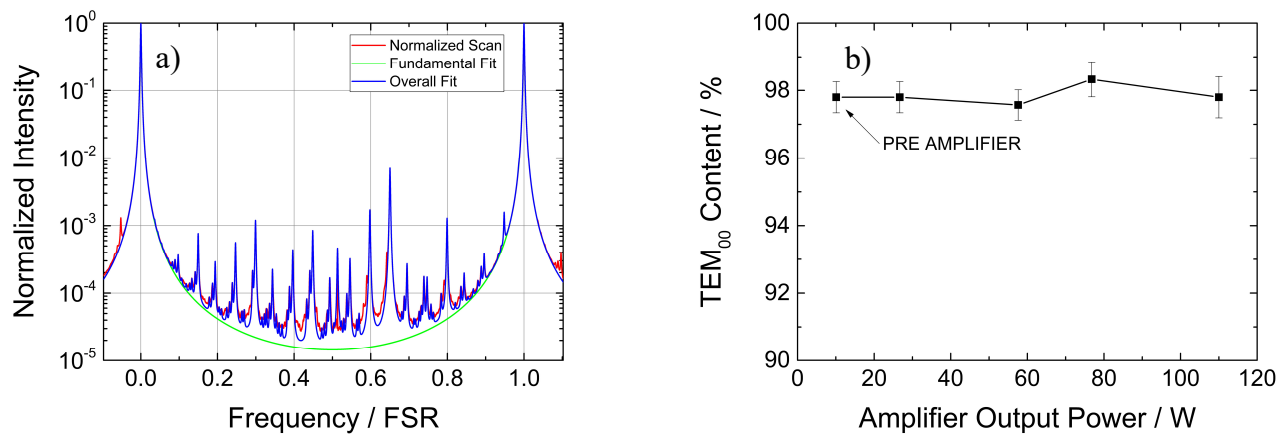

Figure 4: a) Mode scan at $110 \mathrm{~W}$ output power. The normalized transmitted intensity through a ring cavity [14] is shown together with a fundamental $\left(\mathrm{TEM}_{00}\right)$ mode fit and a fit including higher order modes. b) The $\mathrm{TEM}_{00}$ mode content at several power levels up to $110 \mathrm{~W}$. The beam contains $2.2 \% \pm 0.6 \%$ higher order modes at $110 \mathrm{~W}$ amplifier output power.

\section{OPTICAL NOISE PROPERTIES}

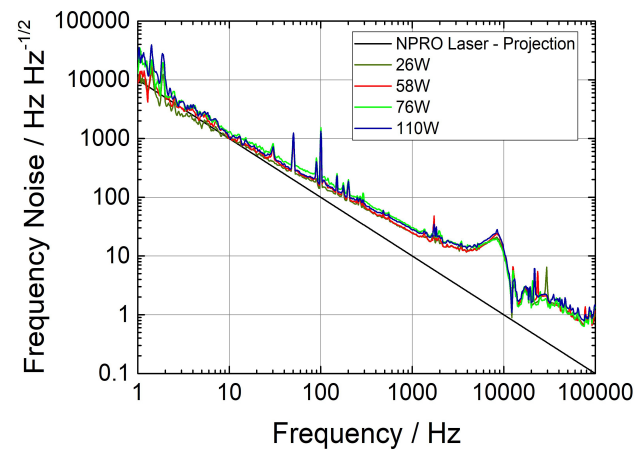

Figure 5: Frequency noise measurements at several mainamplifier module output power levels up to $110 \mathrm{~W}$. The frequency noise remains constant over the entire amplifier output power range. The corresponding linewidth is calculated as $1.7 \mathrm{kHz}$ (over $100 \mathrm{~ms}$ ). The $1 / \mathrm{f}$ NPRO noise characteristic is shown as a reference.
In addition to polarization and beam quality properties of the output beam of the fiber amplifier, several noise properties have been characterized with respect to the typical requirements of GWDs. This includes the relative power noise (RPN), the frequency noise and relative pointing noise. The diagnostic breadboard (DBB) device described in [14] is used to perform these measurements.

Figure 5 shows frequency noise measurements and compares these measurements at several output power levels. The measurements were performed by locking the beam to the ring cavity of the DBB to the fundamental mode. The frequency noise is calculated by evaluating the error signal of the feedback loop [14]. The measurement shows that the frequency noise is nearly unaffected in a range from 1 to $100 \mathrm{kHz}$ at all output power levels. The NPRO frequency noise behavior is plotted as a reference.

The RPN was also analyzed in the frequency range of 1 to $100 \mathrm{kHz}$ up to $110 \mathrm{~W}$. The RPN shows increased noise with higher output power. Because of the transfer 
function from seed to output signal (high pass) and pump to output signal (low pass) the power noise at lower frequencies is determined by the noise of the pump diode. The noise properties are compared to the SSL-system used at the aLIGO Hanford Observatory [15]. The fiber amplifier RPN shows lower noise of up to one order of magnitude in the frequency range from $1 \mathrm{~Hz}$ to $200 \mathrm{~Hz}$. At higher frequencies above $10 \mathrm{kHz}$ the RPN is determined by the seed source, which is the same for the SSL and fiber amplifier system. The pointing noise, shown as RMS value of X and Y measurements remain stable over the amplifier output range. In comparison to the SSL system the pointing noise of the fiber amplifier beam is lower up to approximately $90 \mathrm{~Hz}$. The pointing noise is comparable at higher frequencies up to $10 \mathrm{kHz}$
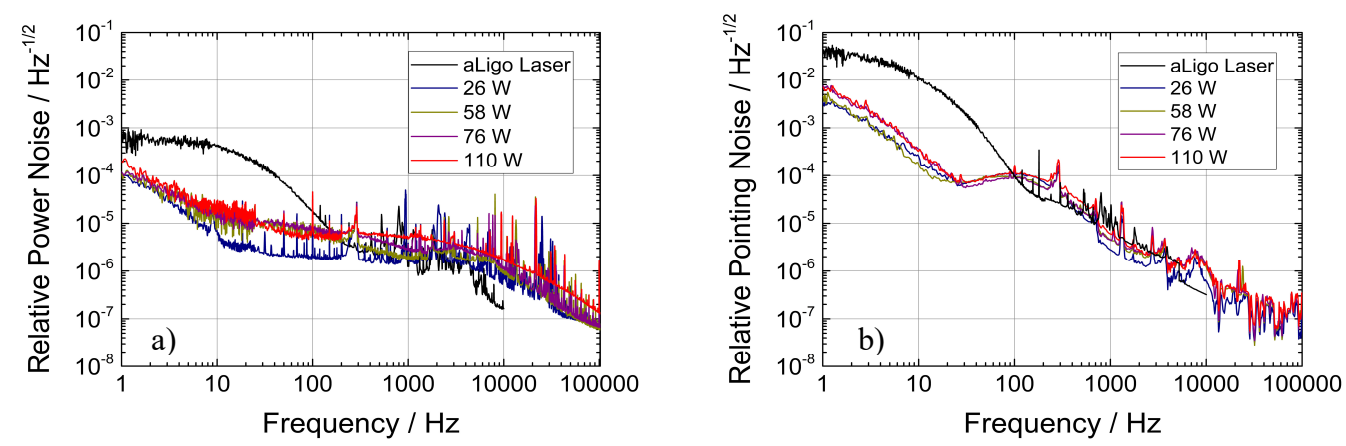

Figure 6: a) RPN measurements up to $110 \mathrm{~W}$ output power of the main-amplifier module. The measurements show degradation with increased power levels. The increased noise level is caused by the pump laser or its current source. The noise measurement of the aLIGO laser source is plotted as reference. The fiber laser shows lower power noise in the range from 1 to $100 \mathrm{~Hz}$ and is comparable at higher frequencies. b) Relative pointing noise measurements up to $110 \mathrm{~W}$ output power of the main-amplifier module. The measurements show degradation with increased power levels. The fiber amplifier output beam shows lower relative beam pointing at frequencies below $90 \mathrm{~Hz}$.

\section{CONCLUSIONS}

Single-frequency fiber amplifiers are very promising laser sources to address the challenging requirements of nextgeneration GWDs. Monolithic systems can be compact, reliable, easy to operate, and simple to maintain. We developed a monolithic LMA $\mathrm{Yb}^{3+}$-doped fiber amplifier using a step-index fiber with a $25 \mu \mathrm{m}$ core. The fiber amplifier system is currently capable of delivering $110 \mathrm{~W}$ of output power with a $\mathrm{TEM}_{00}$ mode content of $97.8 \% \pm 0.6 \%$ and a PER above $21 \mathrm{~dB}$. The frequency noise, RPN and relative pointing noise, which are the important noise properties for the use in GWDs, were also analyzed. The frequency noise at several output power levels was measured and did not show an increase of frequency noise. The relative power and pointing noise were analyzed and show advantages of decreased noise levels at lower frequencies (below $100 \mathrm{~Hz}$ ), compared to the SSL-systems. This system demonstrates that fiber amplifiers are capable of competing and outperform currently used laser sources in GWDs.

\section{ACKNOWLEDGMENTS}

The authors would like to thank Prof. Willke of the Albert-Einstein-Institut Hannover for the successful and on-going cooperation in the field of single-frequency laser systems for GWDs.

Funding was provided by the Max Plank Institute for Gravitational Physics. 


\section{REFERENCES}

[1] B.P. Abbott et al., LIGO Scientific Collaboration and Virgo Collaboration, "Observation of Gravitational Waves from a Binary Hole Merger,” Phys. Rev. Lett. 116 (6), 061102 (2016).

[2] B.P. Abbott et al., LIGO Scientific Collaboration and Virgo Collaboration, "GW170817: Observation of Gravitational Waves from a Binary Neutron Star Inspiral,” Phys. Rev. Lett. 119 (16), 161101 (2017).

[3] B. P. Abbott et al., "Exploring the Sensitivity of Next Generation Gravitational Wave Detectors," Class. Quantum Grav. 34(4), 044001 (2017).

[4] ET Science Team, "Einstein gravitational wave Telescope Conceptual Design Study," available at http://www.et-gw.eu/, Date of access: Jan. 08 2018, ET-0106A-10, (2011).

[5] LIGO Scientific Collaboration et al., Technical Report No. LIGO-E1100716-v6, 24-30 (2012).

[6] M. D. Mermelstein, K. Brar, M. J. Andrejco, A. D. Yablon, M. Fishteyn, C. Headley III, and D. J. DiGiovanni, "All-fiber 194W single-frequency single-mode Yb-doped master-oscillator power amplifier," Proc. SPIE 6873 (2008).

[7] P. Ma, P. Zhou, Y. Ma, R. Su, X. Xu, and Z. Liu, "Single frequency $332 \mathrm{~W}$, linearly polarized Yb-doped all-fiber amplifier with near diffraction-limited beam quality," Appl. Opt. 52, 4854-4857 (2013).

[8] L. Huang, H. Wu, R. Li, L. Li, P. Ma, X. Wang, J. Leng, and P. Zhou, "414 W near-diffraction-limited allfiberized single-frequency polarization-maintained fiber amplifier," Opt. Lett. 42, 1-4 (2017).

[9] T. Theeg, H. Sayinc, J. Neumann, L. Overmeyer, D. Kracht, "Fiber integrated Ytterbium single frequency amplifier with a measured fundamental mode power content of $91 \%$ at 215 W“, Workshop on Specialty Optical Fibers and their Applications (Optical Society of America), paper W3.33 (2013).

[10] M. Wysmolek, C. Ottenhues, T. Pulzer, T. Theeg, H. Sayinc, M. Steinke, U. Morgner, J. Neumann, and D. Kracht, "Microstructured fiber cladding light stripper for kilowatt-class laser systems," Appl. Opt. 57, 6640-6644 (2018).

[11] T. Theeg et al., “All-Fiber Counter-Propagation Pumped Single Frequency Amplifier Stage With $300 \mathrm{~W}$ Output Power", IEEE Photonics Technology Letters 24 (20), 1864-1867 (2012).

[12] T. Theeg et al., "Pump and signal combiner for bi-directional pumping of all-fiber lasers and amplifiers," Opt. Express 20 (27), 28125 (2012).

[13] V. I. Kovalev, R. G. Harrison, "Suppression of stimulated Brillouin scattering in high-power singlefrequency fiber amplifiers", Opt. Lett. 31, 161-163 (2006).

[14] P. Kwee, F. Seifert, B.Willke, K. Danzmann, "Laser beam quality and pointing measurements with an optical resonator,“ Rev. Sci. Instrum. 78 (7), 073103 (2007).

[15] LIGO Scientific Collaboration et al., Technical Report No. LIGO-E1100716-v6, 24-30 (2012). 